\title{
Philosophiques
}

\section{Objectivité et discours chez Hegel}

\section{Jeffrey Reid}

Volume 28, numéro 2, automne 2001

URI : https://id.erudit.org/iderudit/005676ar

DOI : https://doi.org/10.7202/005676ar

Aller au sommaire du numéro

\section{Éditeur(s)}

Société de philosophie du Québec

\section{ISSN}

0316-2923 (imprimé)

1492-1391 (numérique)

Découvrir la revue

\section{Citer cet article}

Reid, J. (2001). Objectivité et discours chez Hegel. Philosophiques, 28(2), 351-367. https://doi.org/10.7202/005676ar

\section{Résumé de l'article}

L'objectivité dont s'occupe la science hégélienne n'est pas celle d'une réalité détachée, mue selon les lois dialectiques, et le discours scientifique n'est pas vrai et objectif parce qu'il serait la réflexion adéquate d'une telle réalité.

L'objectivité scientifique chez Hegel doit être saisie comme le logos , c'est-à-dire le discours de la science elle-même dans son actualité existante. Il s'agit d'un discours qui est son objet et qui est l'objectivité véritable. Ce type de langage est seulement possible s'il est compris comme le moyen terme entre l'être et le penser, c'est-à-dire en termes du syllogisme hégélien et non pas de la prédication. Concrètement, les objets de la science, ou les contenus de l' Encyclopédie , sont eux-mêmes des discours objectifs, c'est-à-dire existants et vrais. Ainsi, par exemple, c'est seulement comme discours (à savoir le contrat, le droit écrit, la constitution et l'histoire) que l'Etat peut constituer un objet/contenu de la science hégélienne. 


\title{
Objectivité et discours chez Hegel
}

\author{
JEFFREY REID \\ Université d'Ottawa \\ jreid@uottawa.ca
}

\begin{abstract}
RÉSUMÉ. - L'objectivité dont s'occupe la science hégélienne n'est pas celle d'une réalité détachée, mue selon les lois dialectiques, et le discours scientifique n'est pas vrai et objectif parce qu'il serait la réflexion adéquate d'une telle réalité. L'objectivité scientifique chez Hegel doit être saisie comme le logos, c'est-à-dire le discours de la science elle-même dans son actualité existante. Il s'agit d'un discours qui est son objet et qui est l'objectivité véritable. Ce type de langage est seulement possible s'il est compris comme le moyen terme entre l'être et le penser, c'est-à-dire en termes du syllogisme hégélien et non pas de la prédication. Concrètement, les objets de la science, ou les contenus de l'Encyclopédie, sont euxmêmes des discours objectifs, c'est-à-dire existants et vrais. Ainsi, par exemple, c'est seulement comme discours (à savoir le contrat, le droit écrit, la constitution et l'histoire) que l'Etat peut constituer un objet/contenu de la science hégélienne.
\end{abstract}

\begin{abstract}
The objectivity that Hegelian science deals with is not a detached reality running on dialectical laws, and scientific discourse is not true and objective because it is the faithful reflection of such a reality. Scientific objectivity, in Hegel, should be understood as logos, as existing scientific discourse. It is a discourse that is its object and is true objectivity. This type of language is only possible if it is grasped as the middle term between mere being and thought, in terms of the Hegelian syllogism rather than predication. Concretely, the objects of science, or the contents of the Encyclopedia, are themselves existing, true discourses. Thus, for example, only as discourse (i.e. the contract, law, constitution, history...) does the State become an object/content of science.
\end{abstract}

Cet article porte sur l'objectivité hégélienne dans son rapport au discours, en tant que celui-ci peut revendiquer le statut de discours scientifique. Si toute analyse épistémologique pertinente se doit de considérer la question du langage, cette perspective est d'autant plus nécessaire là où les notions d'objectivité et de science sont aussi radicalement réinterpretées qu'elles le sont chez Hegel. Pour exprimer notre propos d'une façon lapidaire, il s'agit de montrer que la science hégélienne est objective, c'est-à-dire existante et vraie, dans la mesure où elle est le logos.

Le vrai rapport objectivité-discours est souvent occulté par un certain préjugé qui laisse croire que d'après Hegel l'objectivité se réduit au monde qui se meut selon les lois de la dialectique. Ainsi, pour le dire autrement, l'objectivité entendue comme la nature et comme l'esprit (dans toute son ampleur étatique et historique) ne ferait que suivre inéluctablement le cours d'une logique dialectique interne. Hegel serait surtout un observateur lucide du monde, au point où il a pu en découvrir les articulations intrinsèques, pour ensuite les exprimer dans un langage qui prétend refléter cette logique. 
Cette vision, bien que souvent inavouée, reste courante ${ }^{1}$. En effet, il est facile de souscrire à une proposition comme la suivante : Hegel a compris que la nature et le monde obéissent aux mêmes lois dialectiques que celles qui gèrent la conscience. Cependant, c'est justement cette attribution d'une logique dialectique à l'objectivité ${ }^{2}$ qui engendre un essaim de faux lieux communs sur la philosophie hégélienne : l'idée d'une fin de l'histoire qui trouverait son accomplissement dans l'état prussien, voire dans la démocratie occidentale, l'idée que tout événement serait l'expression d'une logique cachée, déterminante et progressiste, et surtout, l'idée d'un Hegel empiriste.

La notion d'une objectivité mue selon les lois de la logique dialectique vient se répercuter sur le statut scientifique de son discours. Elle fausse, en fait, les conditions de vérité de celui-ci en tant que logos, c'est-à-dire, pour rester à l'intérieur de la pensée de Hegel, en tant que Wissenschaft ou système de la science. Car en comprenant l'objectivité comme auto-déterminante selon des lois dialectiques et naturelles, on aboutit forcément à des absurdités relevées (à contresens) par Kierkegaard et Marx : le sujet philosophant (Hegel), observateur des mouvements dialectiques de l'objectivité, se trouverait à l'extérieur de la réalité qu'il décrit. Son discours se contredirait en tant que Hegel prétend

1. Je ne citerai que quelques expressions de cette perspective. " [Dialectical philosophy] reveals the transitory character of everything and in everything [...] of endless ascendency from the lower to the higher. And dialectical philosophy itself is nothing more than the mere reflection of this process in the thinking brain. " Engels, Friedrich, Ludwig Feuerbach, New York, International Publishers, 1941, p. 12. "La dialectique est la nature propre, véritable des choses elles-mêmes [...] La pensée du philosophe est dialectique parce qu'elle reflète (révèle) le réel qui est dialectique. " Kojève, Alexandre, Introduction à la lecture de Hegel, Paris, Gallimard, 1947, p. 38. « [...] I have made sense of Hegel's idealist metaphysics by considering it as an attempt to show that reality has a rational structure. ", Wartenberg, Thomas, «Hegel's idealism : The logic of conceptuality ", dans, Beiser, F., dir., The Cambridge Companion to Hegel, Cambridge University Press, 1993, p. 114. "[The Phenomenology] is an argument demonstrating that these [historical] experiences are what they are, and follow the order they do, necessarily, for once the life of reason has set in, its course is preappointed by the requirements of the concept. Hence, the period from Augustus to Napoleon [...] is also the display of the logic of a Weltgeist. » De Giovanni, George, «Factual Necessity : On Harris and Weltgeist », The Owl of Minerva 31 :2, 2000, p. 134. De Giovanni attribue cette thèse à H.S. Harris et semble l'accepter, au moins partiellement. Le passage de Harris dont il s'agit est le suivant : " The one advantage I claim to have over previous students is a clear understanding of why the period from Augustus to Napoleon represents the 'logical cycle' of experience [...]. Hegel's Ladder, t.2, Indianapolis, Hacket, 1997, p. 726.

2. Selon cette interprétation, « la logique objective » de Hegel voudrait dire la logique interne aux choses de l'objectivité naturelle. La conscience, ou le penser, ne ferait que refléter cette logique. Par exemple : "The determinations of reflection are mirrored, so to speak, in the internal relations of individual things [...]. [Objective contradiction] is something that inheres in all individual things [...]. Wolff, Michael, "On Hegel's Doctrine of Contradiction ", trad. E. Flynn, E. et Westphal, K., Owl of Minerva 31 :1, 1999, p. 19. Cependant, la science hégélienne ne peut pas se satisfaire d'un tel rapport de réflexion entre l'être objectif et le penser. Le but de cet article est de montrer comment ce rapport est lui-même objectif en tant qu'il est logos, c'està-dire discours scientifique. 
décrire toute la réalité de l'extérieur, d'un point de vue externe. Car en prétendant articuler « tout ce qui est ", il se situerait en dehors de ce " tout ». Le philosophe et son discours constitueraient une sorte de reste gênant à une équation qui ne se laisserait pas résoudre d'une manière satisfaisante.

La vérité d'un tel discours, celui d'un fidèle observateur de la réalité dialectique, ne pourrait que dépendre de l'adéquation exacte entre le discours et le monde qu'il décrit, de la justesse de la réflexion ou la représentation du monde, bref d'une "adequatio rei et intellectus". Or une telle notion de la vérité objective est totalement étrangère au projet scientifique hégélien. Dans ses grandes œuvres telles que la Phénoménologie ${ }^{3}$, la Logique et l'Encyclopédie, aussi bien que dans d'autres écrits moins centraux, Hegel critique toute notion d'une vérité scientifique, c'est-à-dire systématique, qui serait fondée sur l'observation d'une objectivité extérieure. Ce genre de "vérité » se limite à la " certitude ", l'apanage de ce qu'il nomme l'idéalisme subjectif, celui de Jacobi et de Fichte, s'inspirant de Kant et des empiristes britanniques.

Ce qu'il convient surtout de comprendre, et ce qui est central à notre propos, c'est que ce genre de connaissance, que l'on peut qualifier d'empirique, implique une certaine notion du discours qui n'est pas celle de la science hégélienne. Ce qu'il faut également voir, c'est que ces deux notions de discours impliquent deux conceptions opposées de l'objectivité scientifique. Avant de montrer comment l'objectivité et le logos hégéliens se distinguent de l'objectivité et du discours du sujet philosophant observateur du monde, il faudrait s'entendre sur le sens que nous attribuons, dans ce contexte, à " objectivité ". Car la distinction que nous voudrions établir entre les deux ordres de discours se trouve déjà reflétée dans l'ambiguité que peut comporter ce terme.

Le terme « objectivité » comporte communément deux sens bien distincts : d'une part il signifie une réalité concrète qui se tient à distance du sujet ; mais d'autre part " objectivité » signifie la vérité scientifique, dégagée de toute perspective uniquement subjective. Lorsque, dans cet article, nous affirmons que pour Hegel le logos scientifique est l'objectivité véritable, nous disons que pour lui les deux sens se rejoignent. C'est-à-dire que selon Hegel la science est objectivement vraie en tant qu'elle existe comme discours, comme logos qui est lui-même l'objectivité véritable. Ce type de discours n'est pas celui qui reflète ou représente subjectivement une vérité « objective » qui se trouverait ailleurs; elle est cette vérité. Comment une telle notion du logos, du discours scientifique, est-elle possible ?

3. Par exemple «La certitude sensible " de la Phénoménologie de l'esprit, Werke in 20 Bänden, t.3, ed. Moldenhauer, Eva, et Michel, Karl, Frankfurt am Main, Suhrkamp, 1969, p. 82, où Hegel évoque explicitement l'impossibilité qu'a la science de dire le sensible dans sa singularité, et cela malgré les tentatives de certains commentateurs d'affirmer le contraire. Voir Dulckeit, Katherina, "Can Hegel Refer to Particulars ? ", Owl of Minerva 17 :2, pp. 181-194.

4. Dans Foi et Savoir, Hegel attribue à l'influence de Locke et de Hume le fait d'avoir "plongé » l'idéalisme subjectif dans « la finitude et la subjectivité ». Werke in 20 Bänden, t.2, pp. 376-377. 


\section{Le discours scientifique : moyen terme entre l'être et le penser}

L'objectivité du discours scientifique, dans les deux acceptions du mot " objectivité ", est possible seulement en tant que ce langage est conçu comme le moyen terme objectif (existant et vrai) entre deux extrêmes abstraits : l'être, c'est-à-dire le pur étant naturel, et l'activité négatrice, dialectique de la pensée ${ }^{5}$. Évoquer la vérité objective comme le milieu entre l'être (comme étant) et la pensée ne devrait pas surprendre. Car c'est, en fait, la définition la plus classique de la vérité : l'adéquation entre les deux termes, entre la pensée et la réalité, afin de produire des énoncés qui reflètent cette adéquation. Mais chez Hegel, il ne s'agit pas d'une adéquation extérieure, reflétée dans le discours. Le discours de la science est l'existence objective de cette adéquation. Il s'agit donc d'un langage qui ne fait pas que refléter une vérité étrangère, retirée ; il s'agit d'un langage qui EST son objet, qui EST son contenu et qui est, par conséquent, plus objectif et plus vrai que les deux termes extrêmes (l'être et le penser) ${ }^{6}$. Le projet qui consiste à trouver la vérité objective dans la rencontre de l'être et l'activité négatrice ou dialectique de la pensée, se réalise, au plus haut niveau du système de la science dans sa totalité, dans l'articulation même de l'Encyclopédie : la première partie de

5. «Ceux qui ne comprennent rien à la philosophie lèvent les bras au ciel, assurément, quand ils entendent la proposition [Satz] : la pensée est l'être. [Cependant] nous faisons cette présupposition en tant qu'êtres rationnels, qu'êtres pensants. " Encyclopédie des sciences philosophiques, Philosophie de l'esprit, Bourgeois, Bernard, prés., trad., $\mathbb{S} 465$ add.

6. Je cherche toujours en vain cette compréhension de la vérité objective chez les commentateurs. Certains, comme Robert Perkins, décrivent le projet scientifique en termes d'objectivité, sans voir celle-ci comme le résultat de l'être et du penser : "This system achieves objectivity when all perspectives, partial viewpoints, and subjective interests are dialectically mediated into a whole. ", Perkins, Robert, "Perspectivity and Objectivity : A Critical Clue to Hegel's Epistemology », Owl of Minerva, 20 :2,1989, p. 159. Frank Schalow réunit les éléments sans en arriver à l'idée d'un langage qui est lui-même une "synthèse " objective entre l'être et le penser : "In an amorphous way, Hegel formulated the problem of the relation between being and thought, but not so decisively as to view language as having an even greater importance in forming an essential link between the two. " Ainsi Schalow ne dépasse pas l'idée d'un langage qui ne peut que refléter la vérité : "Through its own activity, language makes visible the mediation of opposites, and thereby exemplifies the form of determinateness essential to thought. "Schalow, Frank, "The Question of Being and the Recovery of Language Within Hegelian Thought ", Owl of Minerva, 24 :2,1993, pp. 164, 165. Le débat récent dans la même revue $(30: 1,1998)$ évoque à maintes reprises le rapport entre l'être et le penser dans le contexte du savoir absolu, comme il se présente à la fin de la Phénoménologie. Le fait qu'aucun des participants (S. Lumsden, R. Devos, S. Houlgate, J. Flay, R. Williams, J. Burbidge) ne fait référence à l'incarnation d'un tel rapport dans le langage scientifique hégélien est peut-être révélateur du contexte du débat. En effet, dans la compression conceptuelle et terminale où se présente le savoir absolu à la fin de la Phénoménologie il ne s'agit pas d'articuler la science dans son extension systématique. Néanmoins, dans la mesure où l'on se penche sur la définition hégélienne du savoir absolu comme "l'esprit qui se sait esprit " (Werke in 20 Bänden, t.3, p. 591), il semble indiscutable que l'expression d'un tel savoir ne peut se trouver que dans le verbe de la science elle-même. Comme nous verrons, cette conclusion se révèle d'une façon plus nette dans l'Encyclopédie, où la notion du syllogisme est élaborée. 
l'œuvre, la "Science de la Logique (le penser), et la seconde, la "Philosophie de la Nature " (l'être/l'étant) trouvent leur vérité ou se réalisent dans la troisième partie, la "Philosophie de l'Esprit ", dont le dernier mot est justement "La Philosophie ", le discours scientifique lui-même.

Un bref passage de Hegel peut nous aider à comprendre la notion linguistique dont il s'agit : un langage qui se présente comme "objectivité véritable ». Cette phrase révélatrice se trouve dans l'Encyclopédie, dans le contexte de "l'esprit théorique » où Hegel écrit explicitement sur le langage $\mathrm{du}$ discours scientifique.

Lêtre $[$ Seiende $=$ l'étant $]$, comme nom $[\mathrm{Name}]$ a besoin d'un autre, la signification à partir d'une intelligence représentante, pour constituer la chose [Sache], l'objectivité véritable ${ }^{7}$.

Cette phrase fait explicitement référence à un langage scientifique qui est lui-même " l'objectivité véritable ». Celle-ci se constitue à partir de deux termes extrêmes : l'un est "l'intelligence représentante » (une forme du penser) et l'autre est ce qui est appelé « le nom ». Ce dernier n'est rien d'autre qu'un pur signe linguistique, dépourvu de toute signification intrinsèque. Or le nom ou le signe est un pur étant naturel, c'est-à-dire, selon l'expression de Hegel, " une réalité extérieure ».

Les noms en tant que tels [sont] des réalités extérieures dépourvues de sens pour elle-mêmes, qui n'ont de signification que comme signes ${ }^{8}$.

Autrement dit, le «nom » ou le «signe » est de l'ordre de l'être arbitraire, de l'étant vide et naturel, en attente du sens qui peut lui être attribué, tout comme un nom propre peut s'appliquer à n'importe quel individu. Les " noms " sont ainsi des " mots dépourvus de sens ${ }^{9}$. En revanche, lorsque le « nom », comme pur signe naturel, est pénétré (nié) par une intelligence (le penser), il peut dorénavant constituer un " mot » au sein du discours scientifique, objectif. Les mots du discours scientifique sont remplis du sens apporté, dans le contexte de l'esprit théorique, par "l'intelligence représentante ». Ce contenu significatif constitue la teneur (Gehalt) du discours, lequel est désormais, selon l'expression même de Hegel, «l'objectivité véritable ». Le mot du discours scientifique est véritablement la chose, mais à condition que celle-ci soit de l'ordre de la «Sache» et non plus entendue comme simple $"$ Ding ${ }^{10}$.

7. Bourgeois, trad., Encyclopédie $\$ 464$.

8. Ibid., $\$ 459$.

9. Ibid., $\$ 463$.

10. Les deux termes peuvent se traduire par « chose ", mais en "Sache " il s'agit d'une affaire humaine tandis que le "Ding " est une chose naturelle. Dans la Science de la Logique, la "Sache " surgit sous le titre : "L'émergence de la chose (Sache) en existence ", à l'intérieur de la « Doctrine de l'essence ». L'acte de signification est l'acte de négation par le penser où l'être naturel du signe est nié pour faire de l'essence. L'être meurt (geht zu Grund) pour devenir essence comme ayant été (gewesen). 
Ce qu'il faut surtout retenir c'est que " la chose [die Sache], l'objectivité véritable ", reste langage, mais un langage qui détient plus de vérité et d'objectivité que les signes vides avec lesquels nous avons commencé. Car ce langage réunit la simple réalité extérieure du signe linguistique avec la teneur significative apportée par le penser. À travers le passage du "nom » au " mot ", qui résulte de la rencontre pénétrante entre l'être et le penser, on atteint un niveau d'objectivité plus haut, qui constitue une sorte de "seconde nature ${ }^{11}$, plus riche et plus " sensée » que celle de l'objectivité contingente, pauvre et arbitraire du simple signe.

Bien que le discours "théorique " que nous venons d'évoquer ne doive pas se confondre avec le discours scientifique du système dans son ensemble, il faut voir le premier, à l'intérieur du système scientifique, comme déjà nanti d'un degré d'objectivité et de vérité plus élevé que le langage constitué de signes naturels et arbitraires. Car, en effet, un tel discours se révélerait purement subjectif. Autrement dit, lorsque le langage est constitué de signes vides et naturels, ceux-ci peuvent recevoir n'importe quelle signification qu'un sujet leur impose, et cela de la façon le plus arbitraire qui soit. L'objectivité du discours théorique à l'intérieur du système scientifique s'en distingue déjà par son contenu (Gehalt), lequel ne peut plus être considéré comme arbitraire.

Au niveau théorique, où se trouve la discussion sur le «nom » comme signe linguistique, nous pouvons supposer que nous avons affaire aux sciences de la nature, ou plutôt aux textes qui constituent celles-ci aux yeux de Hegel. C'est-à-dire que le contenu de la philosophie de la nature chez Hegel n'est pas la nature elle-même, mais les discours théoriques de scientifiques qui l'ont déjà pénétrée et niée avec leur penser. Ces discours théoriques, nous dit Hegel, sont plus objectifs que la nature brute. Ils sont, eux-mêmes, déjà pleins de contenu.

Ainsi on voit que la « Philosophie de la nature » est lardée de références aux ouvrages scientifiques de l'époque ${ }^{12}$, lesquels doivent être pris, donc, comme autant de discours relativement objectifs. La science dans sa totalité, à savoir le logos, se configure à partir des discours relativement objectifs qui la constituent. Nous écrivons " relativement » parce que la science ellemême, entendue comme "un ensemble systématique de sciences qui sont pleines de contenu ${ }^{13}$, est nécessairement plus objective que les discours qui

11. Peperzak, Adrian, "Second Nature : Place and Significance of Objective Sprit in Hegel's Encyclopedia ", Owl of Minerva, 27 :1, 1995, pp. 51-66.

12. L'article de John Burbidge, " Hegel on Galvanism », dans Collins, Ardis, dir., Hegel on the Modern World, Albany, SUNY, 1995, pp. 111 - 124, montre bien comment Hegel s'appuie sur les écrits de son époque afin d'élaborer la théorie du galvanisme que l'on trouve au paragraphe 330 de l'Encyclopédie. Par surcroît, Burbidge explique pourquoi Hegel préfère la théorie de Pohl à celle de Berzelius : « (...) from the Logic, philosophy has criteria to anticipate, in general, what kind of explanation will be successful (...)» p. 122 .

13. « Rapport à Niethammer », Werke in 20 Bänden, t.4, p. 411. 
la forment. Pour le dire autrement, " les sciences pleines de contenu », en tant que discours objectifs, constituent à leur tour le contenu de la science hégélienne dans sa totalité et lui confèrent son objectivité propre, ou selon une autre perspective, " la représentation doit [...] être amenée au-delà d'ellemême par la philosophie ${ }^{14}$.

Les sciences théoriques de la nature ne forment qu'un volet du système hégélien. Selon notre argument, les autres contenus du système doivent également se présenter comme des discours objectifs, et cela au même titre que le discours théorique évoqué ci-dessus. C'est-à-dire que les autres contenus de la science hégélienne doivent eux aussi se présenter comme des discours objectifs, le résultat existant d'une " compénétration » de l'être et du penser. Nous voudrions donc montrer, bien que ce soit forcément de façon sommaire, comment une telle notion d'un langage scientifique s'applique également aux autres contenus du système. Cependant, il faudrait auparavant examiner une dimension linguistique du logos hégélien qui va nous permettre de mieux comprendre sa conception d'un langage qui puise sa propre objectivité dans l'incorporation de contenus objectifs. Bref, il faut considérer une notion linguistique hégélienne qui n'est guère considérée comme telle par les commentateurs qui se penchent sur le langage chez Hegel. Il s'agit du syllogisme. Ce concept clef est essentiel à toute compréhension de la saisie hégélienne du discours, et partant, de l'objectivité scientifique. Il permet de comprendre comment un langage apparemment commun, avec un vocabulaire courant et une grammaire prédicative peut atteindre à l'expression du logos dans sa signification la plus systématique et vraie.

\section{Le discours scientifique : de la prédication au syllogisme}

L'objectivité véritable, ce que Hegel appelle die Sache, doit se comprendre en termes de syllogisme ${ }^{15}$ et non pas en termes de prédication, ou en termes de " jugement », pour employer le vocabulaire hégélien. Car seul le syllogisme est capable de produire un contenu plus riche, plus objectif et plus vrai que les deux extrêmes, c'est-à-dire les deux pôles de la science hégélienne : l'être et le penser. C'est seulement ainsi que le contenu du discours scientifique peut se présenter comme "un moyen terme ».

14. Encyclopédie, $1^{\text {re }}$ édition, $\$ 2$, citée par Biard, Buvat, Kervegan et al dans L'introduction à la science de la logique de Hegel, "L'être ", Paris, Aubier, 1981, p. 11.

15. Ce que Hegel entend par "syllogisme » est à l'opposé de ce que veut dire le terme au sein de la logique aristotélicienne. Chez Hegel la structure logique du syllogisme représente la possibilité de la science de se donner un contenu. " Tous les hommes sont mortels ; Caius est un homme, donc il est mortel. On se trouve aussitôt pris d'ennui lorsqu'on entend dérouler un tel syllogisme. " La Science de la logique, Labarrière/Jarczyk, trad., "La doctrine du concept ", p. 160. Werke in 20 Bänden, t.6, p. 358. Voir également Ibid., t.2, p. 541. 
Même les études les plus poussées du langage hégélien, telles que celles de Jere Surber et de John McCumber, semblent hésiter à voir dans le syllogisme la suite de considérations sur l'acte de prédication. Surber, par exemple, dans son analyse de "la phrase spéculative [der Spekulative Satz] ", notion qu'il trouve dans la préface à la Phénoménologie, insiste sur "la structure dialectique de la proposition » et explique comment la phrase spéculative " reflète le fait que pour Hegel la conscience est elle-même une activité dialectique ${ }^{16}$. Autrement dit, il semble que lorsqu'on reste au niveau d'une analyse de l'acte de prédication ${ }^{17}$ en termes de son "activité dialectique ", on ne parvient pas au-delà d'un rapport de " réflexion ", où le langage ne peut fournir qu'une analogie subjectivement observée ou arbitraire de l'activité du penser. Cette dimension arbitraire, qui nous semble tout à fait opposée au projet scientifique de Hegel, se révèle dans l'énoncé de Surber où " la même phrase devient spéculative grâce à la manière selon laquelle nous la comprenons et réfléchissons sur elle ${ }^{18}$. En rester à une telle notion du discours hégélien reviendrait à affirmer que celui-ci n'est rien d'autre qu'une réflexion plus ou moins précise de la pensée. Ainsi la vérité dépendrait entièrement des jugements arbitraires d'un sujet philosophant qui prétendrait décrire " tout ce qui est ». Bref, on rencontre les mêmes contradictions épistémologiques signalées au début de cet article.

En restant au niveau d'une analyse de l'acte de prédication, au lieu de voir dans le syllogisme hégélien la possibilité d'un discours qui est le moyen terme objectif entre l'être et le penser, John McCumber se heurte au même problème que Surber. Chez McCumber ce qui est sens (ou le rapport entre le penser et le langage) est maintenant saisi comme le jugement arbitraire du public, entendu comme une communauté de référence linguistique, à la Wittgenstein ${ }^{19}$. Chez ces deux commentateurs, on voit comment la vérité objective reste très problématique lorsqu'une distance de réflexion est maintenue entre le langage de la science et le penser, lorsque la relation entre les deux est purement analogique. Ce problème est inhérent à toute analyse du discours hégélien en termes de prédication seulement, même si celle-ci est comprise " dialectiquement ». C'est pour cette raison que Surber et McCumber arrivent tous deux à des affirmations surprenantes concernant la nature de la vérité chez Hegel. Par exemple, McCumber affirme que ce n'est pas, en fait, la vérité que la science cherche à exprimer mais «la noblesse ${ }^{20}$. Lorsque

16. Surber, Jere Paul, « Hegel's Speculative Sentence », Hegel Studien, 10, 1975, p. 222. Je traduis de l'anglais.

17. C'est-à-dire selon la forme de la simple proposition " Ceci est cela ", où l'on attribue un prédicat au sujet.

18. Surber, "Hegel's Speculative ", p. 228.

19. " [F]or Wittgenstein then (and Hegel), meaning is public, intrinsically. " McCumber, John, In the Company of Words, Hegel, Language and Systematic Philosophy, Evanston: Northwest University Press, 1993, p. 260.

20. Ibid., p. 227. 
Surber tente d'élargir l'explication dialectique de la prédication jusqu'à une théorie générale du logos hégélien, il arrive à la conclusion tragique que la pensée « ne peut jamais s'épuiser dans une [...] formulation linguistique particulière $»^{21}$. Une telle affirmation semble nier la science hégélienne dans son projet même, à savoir l'élaboration d'un discours scientifique ultime.

La tendance critique qui consiste à considérer le verbe hégélien comme la réflexion analogique du penser peut parfois revêtir une forme d'apparence profonde, voire ésotérique où résonne un écho néoplatonicien. De tels énoncés semblent défier toute tentative d'analyse de la part du lecteur et échappent ainsi à toute compréhension véritable. Dans ce contexte, où le discours est compris comme l'adéquation avec le penser (sans saisir le discours comme moyen terme objectif entre le penser et l'être), le logos se voit doté d'un auto-mouvement parallèle au mouvement que l'on attribue au penser : les deux se meuvent selon les mêmes lois dialectiques. À titre d'exemple nous citons quelques phrases de l'Introduction à la lecture de la Science de la logique où il est explicitement question du discours philosophique. "C'est cela qui revient à étudier la nature de la discursivité spéculative; non pas ce que Hegel " veut dire", mais comment peut se déployer un tel discours nouveau ${ }^{22}$. "Prendre à la lettre le projet hégélien, c'est essayer de voir comment fonctionne ce discours, comment il engendre ou produit d'un même mouvement ses catégories et ses objets ${ }^{23}$. De telles affirmations semblent se présenter comme des noyaux les plus élémentaires de la pensée hégélienne, lesquels résistent à toute analyse.

Ainsi, de la même manière que l'on peut comprendre le discours hégélien comme le reflet de l'être qui se meut selon les lois dialectiques, on peut d'une façon aussi unilatérale considérer le logos hégélien comme le reflet de l'activité dialectique de la pensée. Les deux perspectives sont fausses dans la mesure où elles comprennent le discours philosophique comme une adéquation analogique dont la vérité ne peut que dépendre de la fidélité de cette représentation. Les deux perspectives ne voient pas le langage de la science pour ce qu'il est, à savoir le moyen terme objectif du syllogisme dont les extrêmes sont et l'être et le penser ${ }^{24}$.

Par ailleurs, voir le logos en termes d'une adéquation analogique avec le penser ou avec l'être laisse surgir le problème de l'auteur de l'œuvre, que nous avons signalé plus haut. Si un individu nommé Hegel a bel et bien écrit un livre qui s'appelle l'Encyclopédie des Sciences Philosophiques, comment

21. Surber, «Hegel's Speculative Sentence », p. 228.

22. Biard, Buvat, Kervegan et al, Introduction à la lecture de la Science de la logique de Hegel, p. 12. Je souligne.

23. Ibid. Je souligne.

24. Les auteurs de l'Introduction à la lecture de la Science de la Logique de Hegel comprennent l'esprit comme "l'unité synthétique de la pensée et du réel », et cette unité en termes d'une " organisation discursive ", mais sans voir comment l'esprit en vient à constituer un contenu du discours scientifique. 
réconcilier l'idée d'un discours qui "se déploie » avec la réalité d'un philosophe qui l'a rédigé ? Car " [P]rendre à la lettre le discours scandaleux où s'expose un penser qui prétend, à ce qu'il paraît, régenter la totalité du réel, n'est pas un simple paradoxe ${ }^{25}$.

L'exégèse de la phrase spéculative dans la Phénoménologie est reprise dans la Logique, mais dans un contexte où le jugement (c'est-à-dire la prédication) se présente comme la séparation initiale entre la première instance du concept, comme un tout immédiat, et le syllogisme pleinement développé qui s'articule en moments du singulier, du particulier et de l'universel. En effet, le jugement est compris étymologiquement comme un "Ur-teilen », la division originelle nécessaire pour que le concept puisse se configurer comme un tout médiatisé. Cependant, la définition de la prédication en termes de division laisse peu de place au contenu, et dans la Logique Hegel traite spécifiquement de ce problème ${ }^{26}$. Le contenu essentiel (das Gehalt) ne peut se trouver ni dans le sujet, ni dans le prédicat, qui sont liés d'une façon purement arbitraire, mais se trouve dans la copule, c'est-à-dire dans le « est » qui relie les deux extrêmes de l'affirmation. Ainsi la copule devient " l'unité remplie et déterminée du sujet et du prédicat, comme leur concept ${ }^{27}$. Lorsque la copule se présente de cette manière, nous dit Hegel dans la Logique, le jugement (la prédication) devient syllogisme : "Par cet emplissement de la copule, le jugement est parvenu au syllogisme $»^{28}$.

25. Ibid., p. 13. Ce problème se présente d'une façon tranchante chez Gérard Lebrun : «Tout philosophe, c'est connu, est dogmatique par ce qu'il doit présupposer. Mais c'est autre chose qu'on entend, lorsqu'on parle d'un auteur qui entendait abolir tout présupposé : la certitude ultra-dogmatique d'habiter la Vérité enfin accomplie, de fermer l'Histoire et de pouvoir parcourir du regard du propriétaire toutes les formes culturelles passées et présentes. " Lebrun, Gérard, La patience du Concept, Essai sur le Discours hégélien, Paris, Gallimard, 1972, p. 11. Il est significatif, nous le verrons, que la citation de L'introduction à la lecture... et celle de Lebrun ne distinguent pas entre ce qui est "la totalité du réel », dans le premier cas, ou " toutes les formes culturelles passées ", dans le second, et ce qui constitue pour Hegel un objet scientifique. Ceci est loin de s'entendre comme tout le réel ou tout ce qui s'est passé.

26. Voir Werke in 20 Bänden, t.6, pp. 302-303. Le jugement (l'énoncé prédicatif) ne peut jamais dépasser la représentation. «Dieu, esprit, nature, ou quoi que ce soit, ne sont par conséquent d'abord, [entendus] comme sujets d'un jugement, que des noms ; ce qu'un sujet est, selon le concept, n'est présent que dans le prédicat. [...] Pour cette raison, c'est à proprement parler la simple représentation qui constitue la signification présupposée du sujet (...) ». Par ailleurs, Surber fait remarquer que Hegel nous demande d'accepter le terme "sujet » à la fois selon son sens grammatical et selon son acception consciente. Dans certains écrits tardifs, tels que son compte rendu des écrits de Solger, Hegel en vient à associer la forme du jugement à la subjectivité ironique, avec ses affirmations contre l'objectivité de la science. "Le jugement représente une tendance négative contre l'objectivité (...) » Werke in 20 Bänden, t.11, p. 233. Voir mon introduction de Hegel — L'ironie romantique, Paris, Vrin, 1997.

27. Werke in 20 Bänden, t.6, p. 309. La Logique, Labarrière et Jarczyk, trad., " La doctrine du concept ", p. 108.

28. Ibid. p. 151. (Werke in 20 Bänden, t.6, p. 351) 
Nous insistons sur ce passage entre le jugement (la prédication) et le syllogisme afin de renforcer notre argument à propos de la nature du discours scientifique chez Hegel, à savoir un langage qui se manifeste comme objectif, vrai et plein de contenu. Le passage de la prédication au syllogisme nous apprend que le " est » de la copule " remplie et déterminée » doit être pris au pied de la lettre, si l'on peut dire, selon toute son extension existentielle. Car la copule est le moyen terme "rempli », par lequel le syllogisme atteint "sa vérité, et est ainsi passé de la subjectivité dans l'objectivité ${ }^{29}$. Autrement dit, le moyen terme est précisément ce que Hegel présente comme le mot plein de contenu à l'intérieur du discours scientifique, à savoir "la chose $\left[\right.$ die Sache] $»$ ou «l'objectivité véritable ${ }^{30}$.

Lorsque l'on ne comprend pas les écrits sur le syllogisme comme le développement des considérations hégéliennes sur la forme prédicative et la phrase spéculative on reste ancré au niveau formel d'interprétation. Et toute discussion de la notion hégélienne du langage scientifique exige une analyse qui dépasse le strictement formel pour rejoindre une discussion du contenu. Une telle perspective est largement étrangère à l'analyse linguistique comme elle se pratique aujourd'hui, ce qui explique peut-être la tendance à éviter les considérations de contenu lorsqu'il s'agit d'étudier le langage chez Hegel. Dans les sections suivantes, nous voudrions examiner des exemples susceptibles de montrer comment le discours scientifique hégélien peut incorporer un contenu objectif. La science tire sa propre objectivité de celle de son contenu.

Le contenu de la science, dans sa totalité, n'a rien de mystérieux ; c'est tout simplement le contenu de l'Encyclopédie : la nature, l'état, l'histoire, l'art et la religion ${ }^{31}$. Selon notre argument, ces contenus qui constituent la science hégélienne doivent être pris comme étant eux-mêmes des discours objectifs. Autrement dit, l'objectivité de la science hégélienne dans sa totalité dépend de l'objectivité de son contenu. Nous n'avons pas, bien évidemment, la possibilité d'examiner ici, de façon exhaustive, chacun des contenus de la science hégélienne et de montrer comment il répond à une telle notion de discours. Dans un premier temps, nous évoquerons un élément originaire de l'Etat : la propriété. Cet exemple servira à montrer comment une chose bien naturelle (Ding), récupérée dans le langage peut en venir à former une chose (Sache) ou un contenu objectif de la science. À partir de cet exemple, nous aurons un aperçu de comment les autres choses de la science hégélienne (c'est-à-dire la religion, l'art, la nature et la philosophie) sont également, sous forme de discours, des contenus objectifs et vrais du logos. En dernière analyse, il s'agit de démontrer comment l'objectivité véritable, selon Hegel, est le discours scientifique lui-même.

29. Ibid. p. 156. (Werke in 20 Bänden, t.6, p. 354)

30. Bourgeois, trad., Encyclopédie $\$ 464$.

31. La Logique n'est pas un contenu du logos, mais sa grammaire. 


\section{Les discours objectifs, contenus de la science}

Le premier contenu (ou l'objet) de la science hégélienne que nous évoquons est donc la propriété privée, c'est-à-dire ce qui se présente dans l'Encyclopédie comme la première rubrique de "l'Esprit objectif " (L'État) ${ }^{32}$. Selon notre thèse, ce contenu/objet du logos hégélien doit se présenter comme un discours qui est véritablement objectif, sans que cette objectivité dépende d'une vérité qui ne serait qu'une adéquation exacte entre signifié et signifiant. La propriété, comme tout autre contenu de la science, doit se concevoir comme un langage qui est le moyen terme objectif entre l'être et le penser. Évoquer la propriété est particulièrement révélateur car, qu'elle soit une maison, un champ ou un cheval, son objectivité se présente intuitivement comme tout à fait naturelle ou « objective ». Or, c'est précisément à cause de son aspect naturel ou immédiat, qui n'a pas été médiatisée (ou niée) par le penser, que la chose (Ding) de la propriété ne peut pas, comme telle, faire partie du discours scientifique. Il s'agit de montrer que le discours de la propriété est plus objectif que la propriété elle-même, entendue comme simple chose (Ding) naturelle.

En ce qui concerne la propriété, l'idée directrice de Hegel est de voir qu'elle n'est véritablement objective qu'en tant qu'elle disparaît, c'est-à-dire qu'en tant que la propriété passe d'une volonté individuelle à une autre. Le sens de ce passage n'est ni dans l'affirmation subjective de la possession, dans le fait de déclarer de façon purement prédicative que « ceci est mien ", ni dans les simples «noms» ou signes linguisitiques qui représentent immédiatement le bout de terre, la maison, etc. Ces signes sont aussi naturels et donc pauvres que les choses (Dingen) qu'ils reflètent. Le sens scientifique de la propriété, son objectivité véritable, le fait qu'elle puisse devenir une chose au sens de Sache, ne se manifeste que lorsqu'elle est transférée (vendue et achetée) d'une volonté à une autre. Ce sens se manifeste ${ }^{33}$ dans le langage du contrat.

Ainsi Hegel écrit : "L'intériorité de la volonté qui se dessaisit de la propriété et de la volonté qui la reçoit est dans le royaume de la représentation, et la parole est, dans celui-ci, acte et Chose $[\mathrm{Sache}]) »^{34}$. Il faut comprendre le contrat comme un langage ayant une existence objective, "substantielle ${ }^{35}$ et vraie. Cette vérité est la suivante : l'essence de la propriété est de

32. C'est-à-dire l'Etat au sens organique. Ainsi il est la structure vivante de la substance éthique (Sittlichkeit) et comprend aussi bien les institutions étatiques que l'activité économique privée (le système des besoins) et la famille.

33. C'est pourquoi la chose (Sache) se présente, dans la « Doctrine de l'Essence » de la Logique sous le titre "L'émergence de la chose dans l'existence ». Die Sache doit être entendue comme la manifestation objective de l'essence. Je revendique sa nature linguistique chez Hegel, c'est-à-dire son statut de logos au sens à la fois grec (d'un discours raisonné) et chrétien, du Verbe qui engendre le monde.

34. Bourgeois, trad., Encyclopédie $\$ 493$.

35. Ibid. $\$ 494$. 
passer d'une volonté à une autre ; cette essence se manifeste dans les mots du contrat. Ainsi nous comprenons concrètement la signification de l'idée hégélienne selon laquelle l'essence (das Wesen) peut se penser en termes de l'être passé (gewesen) ${ }^{36}$. C'est seulement en tant que l'être purement naturel disparaît (est nié ou médiatisé) dans le passage d'une volonté à une autre que peut surgir l'essence. Celle-ci ne se disperse pas dans "l'informe tumulte des cloches, ou une chaude montée de vapeurs ${ }^{37}$. L'essence de la propriété s'objectifie dans le langage contractuel, entendu comme le moyen terme d'un syllogisme dont les deux extrêmes sont l'être naturel et le penser (ici sous la forme de la volonté $)^{38}$.

Tout comme c'est dans le langage du contrat que la propriété privée vient constituer un objet/contenu de la science, c'est également en tant que discours que les autres expressions de l'esprit objectif parviennent au statut scientifique. De surcroît c'est seulement parce que l'esprit objectif, ou l'État organique, au sens hégélien, est constitué d'objets/contenus qui sont des discours objectifs, qu'il peut en venir à constituer lui-même un objet/contenu scientifique. Pour étayer une telle affirmation il suffirait de montrer que pour la Science les autres expressions dont Hegel va traiter dans son chapitre sur l'esprit objectif sont d'abord de nature linguistique ou textuelle, et ensuite de montrer comment ces textes peuvent se comprendre comme un langage objectif dans le sens que nous avons expliqué, à savoir comme le moyen terme entre l'être naturel et l'activité de la pensée. En ce qui concerne la première partie de cette tâche on pourrait évoquer le fait que, hormis les considérations sur la moralité subjective ${ }^{39}$, l'esprit objectif concerne surtout le droit (écrit), à savoir les articulations de celui-ci au niveau de la personne juridique et de sa propriété, au niveau de l'administration de la justice et ensuite aux niveaux constitutionnel et international. Même les considérations sur l'histoire mondiale doivent se comprendre à l'intérieur d'une

36. Werke in 20 Bänden, t.6, p. 13.

37. Hyppolite, Jean, trad. Phénoménologie de l'esprit, t.1, Paris, Aubier, 1941, p. 183. (Werke in 20 Bänden, t.3, p. 169)

38. Il est intéressant de noter que, selon la théorie de la démonstration d'Aristote, l'essence se trouve dans le moyen terme du syllogisme. Aubenque, Pierre, Le Problème de l'être chez Aristote, Paris, PUF, 1962, p. 482.

39. "La moralité ", qui constitue le moment de la liberté subjective à l'intérieur de l'esprit objectif, se définit par la scission entre la volonté individuelle et la liberté abstraite ou idéale. Le discours de ce moment est également scindé et scindant car c'est le langage du jugement (Urteilung). Ainsi son expression la plus radicale est celle de l'ironie, laquelle s'oppose au discours objectif du logos scientifique. Voir Philosophie du droit \$140. Cependant, comme c'est toujours le cas chez Hegel, ce moment n'est opposé à la science comme totalité organique que pour autant qu'il se fixe et s'isole par rapport au mouvement du tout où le discours de l'individu abstraitement libre serait repris à l'intérieur de la substance éthique. 
réflexion sur le droit constitutionnel car "l'histoire des constitutions est l'histoire du développement de ces états ${ }^{40}$.

C'est pourquoi, au dire de Hegel, " la véritable liberté », celle qui est le but de l'histoire, se trouve d'abord dans le droit écrit et ses institutions : "l'obéissance envers la loi et les institutions politiques légales [...] est ellemême la véritable liberté " ${ }^{41}$. Dans ce sens les considérations hégéliennes sur l'histoire mondiale peuvent se comprendre à partir d'une histoire des constitutions politiques; il ne s'agit pas d'une histoire événementielle. L'idée que le contenu de la science se trouve dans des textes qui doivent être pris comme objectivement vrais en eux-mêmes, selon la notion linguistique que nous sommes en train de développer, explique également le fait que chez Hegel la discussion proprement historique s'accompagne d'une discussion historiographique, par exemple, comme c'est le cas au commencement des Leçons sur la philosophie de l'histoire.

La tendance à ne pas voir ce qui constitue la matière véritable de la philosophie hégélienne de l'histoire donne lieu aux malentendus que nous avons signalés plus haut. Ceux-ci s'enracinent dans l'idée erronée que les événements de l'histoire, c'est-à-dire tous les événements actuels qui sont « en passe » de constituer le passé, de devenir l'histoire, sont déterminés par une logique cachée, inhérente et finalement naturelle. Hegel, le fidèle observateur de ce processus en serait le rapporteur. Mais l'histoire, selon Hegel, n'est pas une suite d'événements. En tant qu'elle peut constituer un objet/contenu de la science, la matière brute de l'histoire doit d'abord être sursumée (aufgehoben) dans un ensemble de discours et de textes, de témoignages, de récits, de lois et de constitutions. Bref, Hegel n'observait pas les événements, il lisait des textes.

Il est peut-être moins évident de voir comment d'autres manifestations étatiques, qui ne semblent pas se fonder sur l'aspect textuel du droit, peuvent constituer des objets scientifiques en tant que discours objectifs. Quels discours objectifs, à part le contrat, pourraient constituer le contenu scientifique fondateur du système des besoins, du libre échange des biens à l'intérieur de la société civile ? Quel discours objectif pourrait former le contenu de la famille, comprise comme objet scientifique ? À la première question, nous pourrions évoquer l'influence fondatrice des textes des Lumières écossaises

40. Bourgeois, trad., Encyclopédie \$527. L'expression « ce qui est rationnel est réel et ce qui est réel est rationnel ", laquelle ne fait qu'exprimer l'union du penser avec l'être, apparaît pour la première fois dans les Leçons de Hegel à Heidelberg (1817-18), dans le contexte d'une discussion sur la constitution (écrite). "Ce qui est rationnel doit se réaliser puisque la constitution est, après tout, son développement. " Citée par Avineri, Schlomo, compte rendu, Owl of Minerva, $16: 2,1985$, p. 203. Par ailleurs, comme on voit dans le paragraphe 549 de l'Encyclopédie, l'histoire mondiale est essentiellement historiographie, dont les expressions objectives, mais pas encore spéculatives, sont 1) l'histoire originelle et 2) l'histoire réflexive. L'histoire elle-même ne devient rationnelle (dialectique) que lorsqu'elle est saisie spéculativement dans la philosophie de l'histoire.

41. Bourgeois, trad., Encyclopédie $\$ 552$. 
sur la notion de la société civile chez Hegel ${ }^{42}$. À la seconde nous pourrions simplement répondre, Antigone.

L'objectivité scientifique de l'État, ce qui fait qu'il peut constituer l'objet, ou le contenu, de la science hégélienne se trouve d'abord dans les différents discours qui le forment : les contrats, le droit, la constitution, les textes historiques, économiques, politiques et classiques. C'est en tant que ces discours sont compris comme les moyens termes objectifs, entre l'activité de la pensée et l'être naturel, que l'Esprit objectif (c'est-à-dire l'État) peut, toujours comme texte, revendiquer une vérité objective à l'intérieur de la science hégélienne.

L'État ne forme qu'un des contenus principaux de la science. Il nous est trop tentant de faire allusion, de manière sommaire, aux autres objets de l'Encyclopédie et de proposer comment ceux-ci peuvent également se constituer à partir de discours objectifs.

1) Dans la philosophie de la nature, que nous avons associée au discours théorique, Hegel cite une abondance de sources secondaires, à savoir des textes auxquels il accorde le statut de vérité objective ${ }^{43}$.

2) Dans sa philosophie de l'art, l'objectivité du discours scientifique se révèle dans la supériorité que Hegel accorde aux expressions verbales, à l'opéra plutôt qu'à la musique sans paroles, dans sa préférence pour «ce langage supérieur, la tragédie ${ }^{44}$ plutôt que pour la sculpture, ou encore dans la primauté que Hegel accorde à la philosophie de l'art sur l'art lui-même ${ }^{45}$.

3) Dans la philosophie de la religion, les doctrines de la religion chrétienne sont saisies comme des textes qui sont eux-mêmes la vérité objective. Ainsi, «le vrai contenu de la religion se présente d'abord à l'esprit en mots et en lettres », bref, « en mots et écrits » ${ }^{46}$.

42. Waszek, N., The Scottish Enlightenment and Hegel's Account of Civil Society, Dordrecht, Kluwer, 1988.

43. Voir encore l'article cité plus haut de John Burbidge. Par ailleurs, si les recherches de Mesmer sur le somnambulisme et celles de Goethe sur la couleur s'avèrent, selon Hegel, plus vraies que les recherches de Newton, ce n'est certainement pas parce que les premières répondent mieux aux exigences de la science empirique, selon laquelle l'objectivité dépend de la réflexion adéquate, dans le discours, d'une réalité qui se trouverait ailleurs. Comme l'indique Burbidge, dans ses choix de sources scientifiques Hegel privilégie celles où l'on voit le mieux incarnée une forme de pensée spéculative.

44. Hyppolite, trad., Phénoménologie, t.2, p. 246. (Werke in 20 Bänden, t.3, p. 534.)

45. Ce qui est décadent dans l'art romantique c'est précisément sa nature symbolique, le fait que son discours se borne à refléter, de façon nostalgique, une vérité toujours au-delà. Son discours n'est pas lui-même la vérité objective, le moyen terme entre l'être comme simple signe naturel et l'activité de la pensée qui est, dans le contexte absolu où se trouve l'art, l'Idée ellemême, c'est-à-dire Dieu.

46. Préface à La philosophie de la religion de Hinrichs, Werke in 20 Bänden, t.11, p. 44. A l'encontre de l'herméneutique de Schleiermacher, pour qui " toute écriture sainte n'est qu'une mausolée pour la religion " (première édition des Discours sur la Religion de Schleiermacher, Kritische Gesamtausgabe I.2, Peiter, Birkner et al, dir., New York, Berlin, de Gruyter, 1980, p. 242), d'après Hegel les textes religieux ne doivent pas se penser en termes de signifié et signifiant. Les deux pôles, à savoir l'être et le penser, se rejoignent dans l'unité objective des « écrits ». 
4) L'ultime expression du discours objectif de la science, celle que nous évoquons en conclusion, est bien entendu la science elle-même. Qu'est-ce que cette science ? Elle n'est rien d'autre que l'ensemble systématique de ses contenus, de ses objets, à savoir les discours que nous venons d'aborder, pensés spéculativement. La science pense la vérité objective (ou de l'objectivité véritable) de ses propres contenus et se sait vraie et objective. Ce savoir est le discours existant de la science, c'est-à-dire le logos.

\section{L'actualité du discours de la science}

On pourrait répondre que cette idée d'une objectivité véritable qui serait de nature linguistique ou discursive semble bien décharnée, qu'elle semble réduire toute la richesse du monde à une réalité en deux dimensions, à des mots sur une page. Mais une telle objection s'appuie sur une autre notion du langage, du logos, que celle que nous sommes en train de présenter comme le logos hégélien.

Hegel ne nie jamais la richesse du monde ; ses écrits de Berlin ${ }^{47}$ montrent bien qu'il y participait pleinement. Mais pour ce qui est de la science, il faut insister sur le fait qu'il n'est pas question du monde en général. Il s'agit uniquement d'objets scientifiques, c'est-à-dire des objets dignes d'être pris en considération par la science. Ce n'est certainement pas la plume de $\mathrm{Krug}^{48}$ ou n'importe quel autre objet " naturel ", c'est-à-dire arbitraire et singulier. Les objets scientifiques sont les contenus de la science; leurs noms se trouvent dans la table des matières de l'Encyclopédie. Ce sont des discours vrais et objectifs en eux-mêmes.

Cependant, en ce qui concerne le rapport entre le logos scientifique et le monde, il ne faut jamais perdre de vue que l'objectivité même des discours qui constituent le contenu de la science fait en sorte que ceux-ci existent dans le monde. Autrement dit, c'est parce que ce langage est, et parce qu'il est vrai, au sens d'une réalisation objective entre le penser et l'être, que ce langage doit participer au monde. Pour le dire plus concrètement, l'existence objective du langage du contrat fait qu'il est lu et reconnu par les volontés individuelles à l'intérieur de l'État, entendu comme substance éthique (Sittlichkeit). Cela veut dire que les contrats ne sont pas des lettres mortes; ils sont signés, discutés et respectés ou non. De la même façon les lois de la cité et la constitution elle-même sont vécues par les citoyens, d'une manière

47. Pöggeler, O, « Komödie des Lebens - Theorie der Komödie » dans Hegel in Berlin, Staatsbibliothek Preussischer Kulturbesitz in Verbindung mit dem Hegel-Archiv, 1982, p. 79.

48. En 1802 Hegel rédigea un article polémique contre le philosophe Krug qui avait exigé des philosophes spéculatifs de déduire sa plume. L'article de Hegel affirme que la philosophie n'a pas à s'occuper d'objets singuliers, naturels. 
litigieuse ou non, ainsi que, sur une autre échelle, le «Droit public international ${ }^{49}$ incarne l'activité réciproque des États entre eux (c'est-àdire des constitutions, lois, institutions, etc.) et l'histoire mondiale s'avère le discours des discours de cette activité.

Il en va de même pour les autres expressions du langage objectif de la science. Les discours qui constituent l'art participent pleinement à la vie de la cité. Les doctrines religieuses y participent autant que les autres expressions, voire plus, si l'on veut croire aux déclarations comme celle-ci : "la substantialité de la vie éthique elle-même et de l'État est la religion ${ }^{50}$.

Il ne faut pas comprendre par là que c'est sur l'actualité de ces discours, sur le fait qu'ils participent au monde, que se fonde leur objectivité. C'est-àdire qu'il ne faut pas penser que c'est parce que ces discours existent dans le monde qu'ils sont objectifs. En fait, c'est tout à fait le contraire qui est vrai. C'est parce que ces discours sont vrais et objectifs, selon la notion du langage scientifique que nous avons présentée, qu'ils doivent se manifester comme actuels.

Alors, qu'en est-il de l'actualité du discours de la philosophie ellemême, c'est-à-dire de l'Encyclopédie et des autres écrits hégéliens ? En dehors de toute participation à la réalité effective que peuvent avoir ses contenus, quelle actualité peut avoir le logos scientifique lui-même à l'intérieur de la cité ? Une réponse possible et conséquente consiste à rappeler tout simplement que Hegel a passé presque toute sa vie adulte dans l'enseignement, et que presque tous ses textes écrits étaient destinés à servir de manuels pédagogiques à l'intérieur du système éducatif de l'Etat. Bref, l'actualité du discours qu'est la science, entendue comme le logos objectif et vrai, pourrait bien se trouver dans sa destination pédagogique ${ }^{51}$.

49. Voir Philosophie du droit $\$ 330$.

50. Bourgeois, trad., Encyclopédie, $\$ 552$.

51. Il serait difficile d'exagérer l'étendue de cette destination pédagogique. Selon Hegel, l'enseignement de la philosophie spéculative à l'université présuppose l'acquisition de son contenu, et ensuite l'apprentissage du moment de la négativité dialectique (les paradoxes de Zénon et les antinomies de Kant). Cette formation devait avoir lieu au gymnase. Ainsi l'enseignement de la philosophie (hégélienne) détermine la conception de tout le système éducatif de l'Etat. Voir " Rapport à Niethammer » dans Bourgeois, Bernard, trad., prés., Hegel, Textes pédagogiques, Paris, Vrin, 1990, pp. 142-145. 
